\title{
Analysis of Cellular Microscopy Images by Means of Neighbourhood Graphs
}

\author{
François Angot $\left({ }^{1}\right)$, Abderrahim Elmoataz $\left({ }^{1}\right)$, Marinette Revenu $\left({ }^{1}\right)$ \\ and Paulette Herlin $\left({ }^{2}\right)$ \\ $\left({ }^{1}\right)$ Laboratoire GREYC, UPRESA CNRS 6072, ISMRA, 6 Bd Maréchal Juin, 14050 Caen Cedex, \\ France \\ $\left({ }^{2}\right)$ Service d'Anatomie Pathologique, Centre François Baclesse, Route de Lion sur Mer, \\ 14021 Caen Cedex, France
}

\begin{abstract}
Résumé. - Différents problèmes de traitement et d'analyse d'images, en deux ou trois dimensions, peuvent être modélisés, traités et résolus par les techniques et les outils qu'offre la théorie des graphes. Dans cet article, une représentation des images basée sur les graphes est proposée : une structure de données ainsi que des méthodes de valuation et de pondération, de fusion de sommets. Pour démontrer l'intérêt d'une telle approche, nous présentons deux applications 2D concernant l'étude d'images de microscopie cellulaire.
\end{abstract}

\begin{abstract}
Many image processing and image analysis problems, in two or three dimensions, can be addressed and solved by methods and tools developed within the graph theory. In this paper, an image representation based on graphs is proposed: a data structure, together with methods for attributing and weighting the graph, methods to merge nodes, etc. In order to demonstrate the interest of the approach, two applications dealing with 2D-images of cellular microscopy are described and discussed.
\end{abstract}

\section{Introduction}

Many image processing and image analysis problems can be addressed and solved by methods and tools developed within the neighbourhood graph theory. It is particularly the case for two kinds of problems:

- hierarchical segmentation problems solved by bottom-up strategies [1], when starting with an over-segmented image and building a graph representing each region with both intrinsic and relational attributes [2]; region-merging and region-growing methods can be replaced by graph contractions and calculations of new attributes,

- problems dealing with a population of objects and their neighbourhood relations [3], as in several histological applications; in cellular sociology [4], relations between the functional 
state of the cells and their spatial distribution within the tissue are studied; in the quantitative analysis of the tissue architecture, the distribution of one kind of cells within another is quantified [5].

This paper presents a segmentation and characterization approach based on the graph theory, and its application to problems studied in collaboration with the François Baclesse Cancerresearch centre of Caen (CFB).

\section{Neighbourhood Graphs}

A graph $\mathrm{G}$ is a mathematical object defined by two inter-connected sets: a set $\mathrm{V}$ of points, and a subset $\mathrm{E}$ of $\mathrm{V} \times \mathrm{V}$ [6]. The points of $\mathrm{V}$ are the vertices of the graph, the elements of $\mathrm{E}$ are called the arcs when being oriented, the edges when non-oriented. One has:

$$
\mathrm{G}=(\mathrm{V}, \mathrm{E}) \text { with } \mathrm{E} \subset \mathrm{V} \times \mathrm{V}: \mathrm{k} \in \mathrm{E} \Leftrightarrow \exists \mathrm{p}, \mathrm{q} \in \mathrm{V} \text { where } \mathrm{k}=(\mathrm{p}, \mathrm{q}),
$$

which can also be written as:

$$
\mathrm{V}=\left\{\mathrm{p}_{i}\right\} \text { and } \mathrm{E}=\left\{\mathrm{k}_{i, j}\right\} \text { where } \mathrm{k}_{i, j}=\left(\mathrm{p}_{i}, \mathrm{p}_{j}\right) .
$$

For a non-oriented graph, the neighbourhood relation between two vertices $\mathrm{p}_{i}$ and $\mathrm{p}_{j}$, extremities of an edge, is an equivalence relation, which is the base of all further processing on graphs.

An image is generally considered as a grid of points, corresponding to grey level values. In a graph-based approach, an image can be seen as a set of values, associated to a method returning the neighbours of a point. One can then compare the vertices of a graph and the points of an image. Edges symbolize the grid. However, whereas for a $2 \mathrm{D}$-image, relationships such as the 4-neighborhood or the 8-neighborhood are deduced from the digitalisation grid, and are consequently fixed, in the case of graphs, the neighbourhood relationships are much more general and independent of the real localization of vertices in space. So, graphs allow to represent $2 \mathrm{D}$-images, as well as 3D-images.

In cellular microscopy images, one has to handle populations of objects: cell nuclei. Obtaining a graph from an image is done through the Voronoi diagram which gives an image tessellation according to the distribution of objects. The dual graph, the Delaunay graph, is a neighbourhood graph of these objects. Figure 1 presents an example of a cytology image and the neighbourhood graph associated to the nuclei distribution.

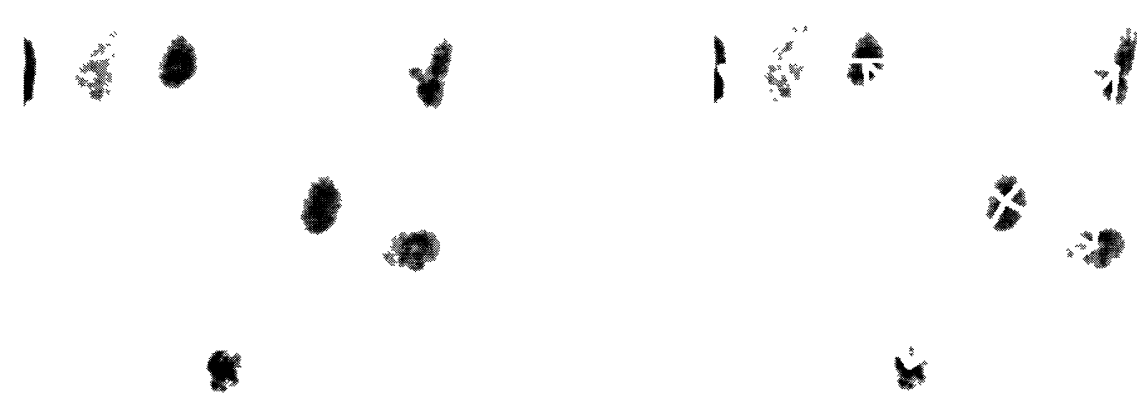

Fig. 1. - Cytology image and neighbourhood graph, associated to the nuclei distribution. 
This image/graph analogy allows to extend several notions defined on images to graphs:

- in images, regions are sets of neighbouring points; in graphs, they form connected-components,

- in images or graphs, paths are series of neighbouring points,

- in graphs, a distance between two points can be defined by counting the number of edges in the path between the two points, whereas in images, it is often calculated by means of a weighted mask on a neighbourhood; this notion of weighted masks can be extended to graphs by means of a weighting function $\mathrm{W}$ on edges that defines a distance between the extremities of edges.

In addition to providing a representation of the organization of objects, graphs allow to handle information about the objects. Through an attributing function $A$, a value characterizing the represented object can also be associated to each vertex, in the same way as a grey level value is associated to a point of an image. This attributing function allows to handle classical objectfeatures such as size, grey level homogeneity, etc.

\section{Implementation of Graph Operators}

In order to process images by means of neighbourhood graphs, a 3-step general strategy has been defined:

- building a graph from an image, for instance, the Delaunay graph deduced from the Voronoi diagram,

- attributing and weighting this graph with suitable functions according to the application,

- rewriting image operators to apply them to graphs; when developing such operators, two kinds of data examinations exist:

- a sequential examination considers the vertices in a pre-defined order; this can be compared to the video-scan of an image,

- an examination of the neighbours of a point as it occurs in growing-based operators: vertices are examined in an order that depends on their neighbourhood and their values.

Our operators on graphs have been developed within the object-oriented environment of the Pandore library [7], where a same operator for different data types can be defined. For example, the thresholding operator can work on a grey level image as well as a region map or a graph.

3.1 Classical Image Processing. - It is simple to develop graph operators analogous to image operators that change the value of a point according to its value and those of its direct neighbours. This is the case for arithmetic operators (ex: addition), logical operators (ex: and, or), filtering operators (ex: blur), derivation operators (ex: gradient), etc.

3.2 Mathematical Morphology. - For a binary or a numerical graph, the same definition of simple morphologic operations (erosion and dilation) or more complex ones (opening) than on images can be used [8]. For instance:

$$
\epsilon_{n}(\mathrm{G})(\mathrm{p})=\min \left\{v(\mathrm{q}) \text { for } \mathrm{q} \in \mathrm{V}_{n}(\mathrm{p})\right\} .
$$

Figure 2 gives examples of morphological operations on a graph, visualized on the associated Voronoi diagram. 

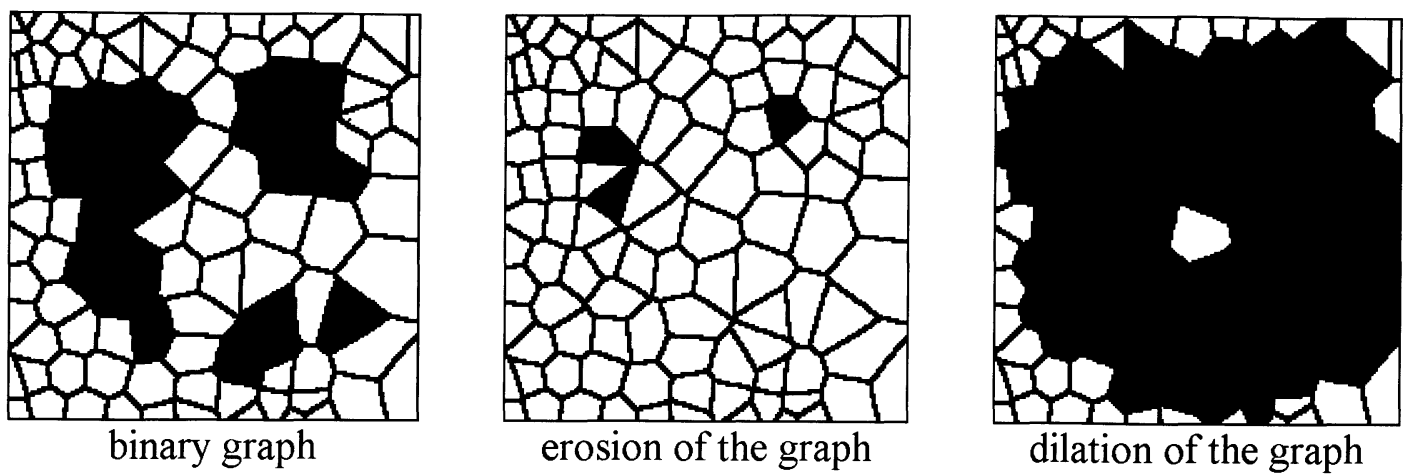

Fig. 2. - Examples of morphological operations on a graph (visualization on the associated Voronoi diagram).

3.3 Operators for Merging Vertices. - Following a bottom-up strategy, several image segmentation problems can be solved by an over-segmentation stage, followed by the merging of some image primitives. When representing the image by a graph, this second stage is done by merging the corresponding vertices.

A general function for merging any two vertices of a graph (and doing all the necessary updating of the graph) was implemented. Then, a Horus operator was defined for each merging criterion, that is in charge of applying this merging function. In general, two vertices will be merged when the weight of the corresponding edge stands between two given thresholds. In complex problems, one often has to perform several merging stages, using different criteria.

3.4 Statistical Characterization. - When considering an image as a population of objects, the aim is not only to segment it, but also to determine one or several values characterizing the population. As each vertex of a graph represents an object, this characterization consists in studying the statistical distribution of the attributes of vertices.

A very commonly used information is the grey level histogram of the points of an image. In the same way, the histogram of any attribute of the vertices of a graph can be built. Moreover, as edges can be weighted by an information of distance between objects, this value distribution can be characterized.

As an extension, a set of attributed points can be characterized by a second order histogram, a cooccurrence matrix $M$ :

$$
M(i, j)=\operatorname{Card}\{\mathrm{k}=(\mathrm{p}, \mathrm{q}) \in \mathrm{A} \text { where } v(\mathrm{p})=i \text { and } v(\mathrm{q})=j\} .
$$

Descriptors such as second-order moment, contrast, entropy of such a matrix, are then calculated on a wide range of object features: shape, orientation, distance from the nearest neighbour, etc.

\section{Applications}

4.1 Hierarchical Segmentation, Application to Cytology Quantification. - The aim of this application is to apply a graph-based segmentation on cytological images, in order to extract nuclei from the background and to separate them (Fig. 3a). The final objective is to characterize the cell nuclei and classify then. As a hierarchical segmentation, according to a bottomup strategy, has already given good results with classical operators, we tried here to simplify 

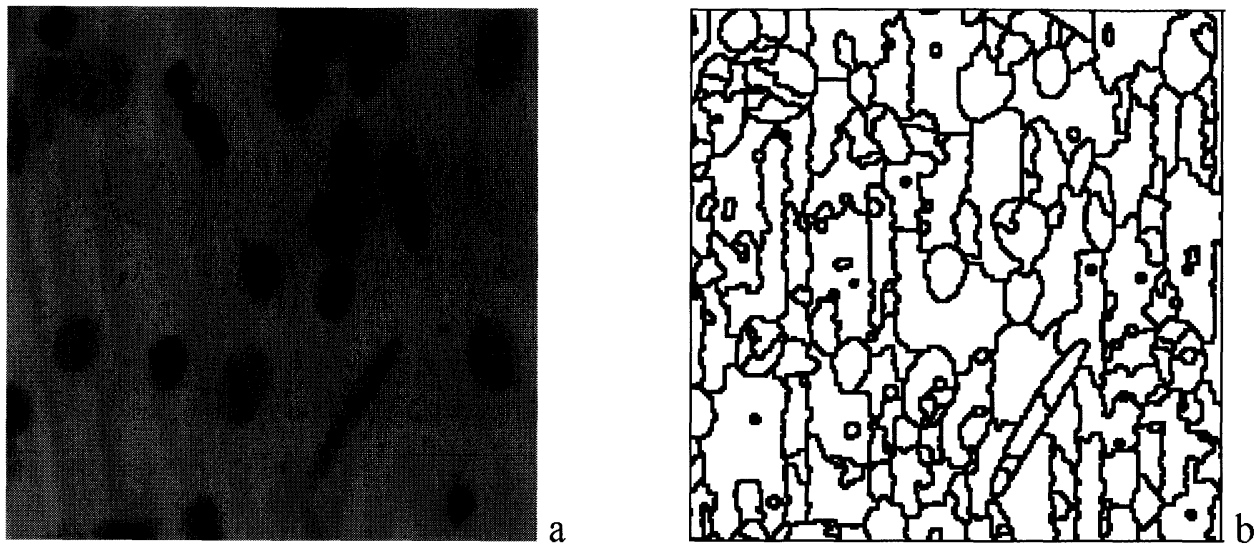

Fig. 3. - Example of a cytology image and initial over-segmentation.

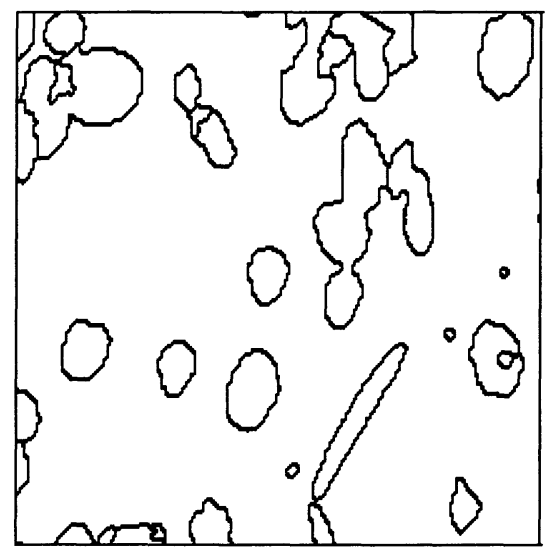

Fig. 4. - Merging according to the mean grey level of the regions.

the processing by using graphs: they are a good way to handle neighbourhood relationships between regions to merge.

The application of a watershed operator on the gradient image, using the regional minima of this gradient image as germs, results in an over-segmentation: the output image contains all nuclei boundaries, but numerous nuclei are still cut into several regions (Fig. 3b).

To improve the initial over-segmentation by merging regions, an adjacency graph is built: a vertex of the graph is associated to each region, and an edge to each boundary. Then, it is simple to weight edges with the difference between the attributes of the corresponding vertices, for instance with the mean grey level (Fig. 4), and to use this weight for deciding whether to merge or not adjacent regions.

4.2 Study of Tissue Architectures. - In the following application, images come from histological sections of tissues, made by the Pathology Department of the CFB of Caen. The aim is to delimit significant nuclei clusters. In this case, graphs allow to take nuclei proximity into account, without using the size of nuclei in the calculus of the inter-nuclei distance. 

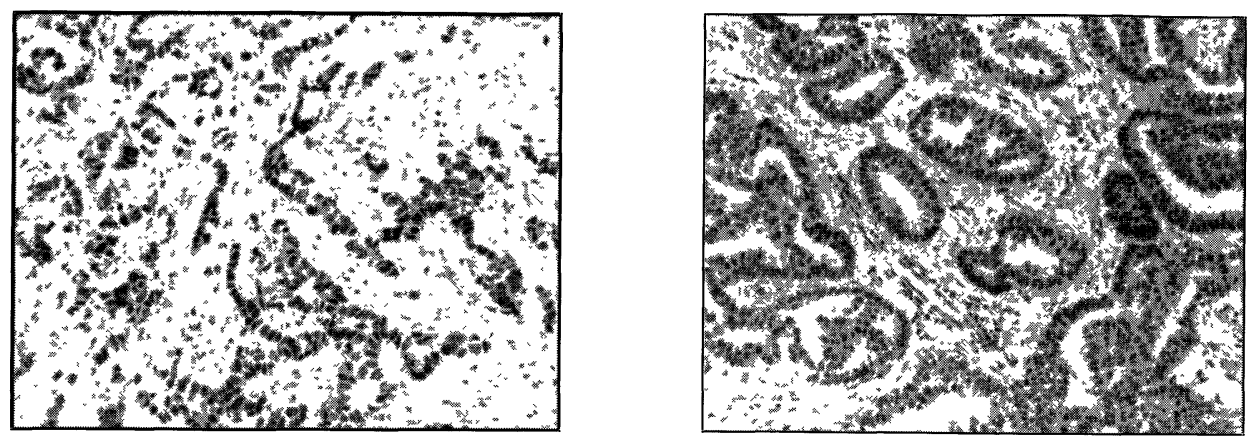

Fig. 5. - Two types of tissue architectures in cases of breast cancer.
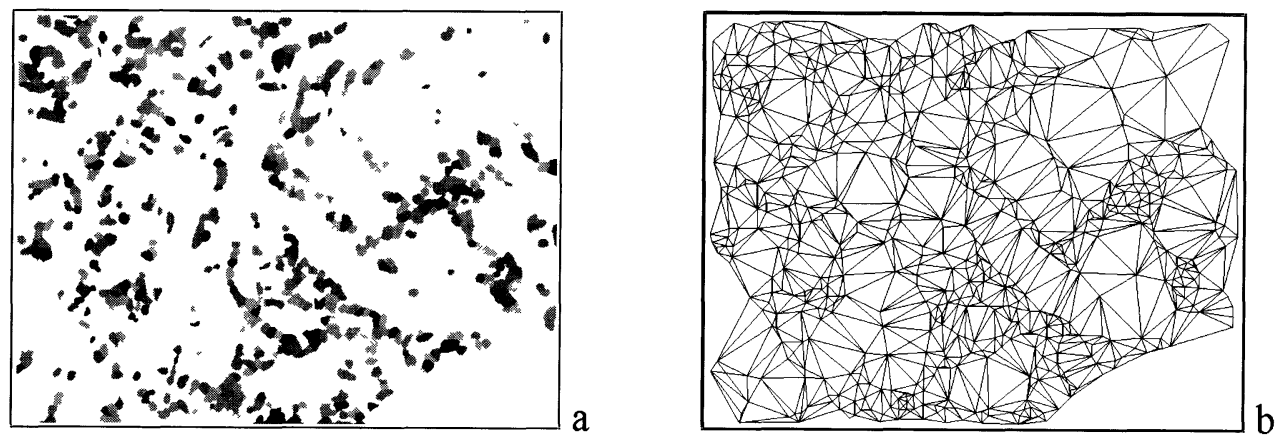

Fig. 6. - Initial segmentation of the nuclei and neighbourhood graph.

Histological sections of mammal tissue, seen through $\times 33$ magnification, are digitized into colour images of $768 \times 576$ pixels. The green component gives a grey level image in which all nuclei (blue and brown) are well contrasted.

To illustrate the wide variability of processed images, Figure 5 presents two types of tissue architectures in cases of breast cancer.

First, nuclei are extracted from the background (Fig. 6). This is done by a watershed segmentation of the image, using its regional minima as germs. After removing "small" noisy regions, the Voronoi diagram gives the neighbourhood graph (Fig. 6b).

A cluster being defined as a set of "close" nuclei, the edges of the graph are weighted by the distance between nuclei, in order to segment the graph on this criterion. Then, "small" clusters are eliminated. The final result is a binary image showing the boundaries of clusters (Fig. 7a), but one has also built a structure describing the internal organization of the nuclei groups (Fig. 7b).

By changing parameter values of the operators, this approach can be applied to other classes of images, to study, for example, the papillary ascension in oesophageal epithelium (Fig. 8).

\section{Conclusions}

In this paper, we tried to show the major advantages of using neighbourhood graphs for image processing and analysis. Thanks to promising results in the detection of nuclei clusters in histological sections, we are now considering how to characterize these clusters. This will be done by further processing on the sub-graphs which represent the internal architecture of clusters. Finally, 

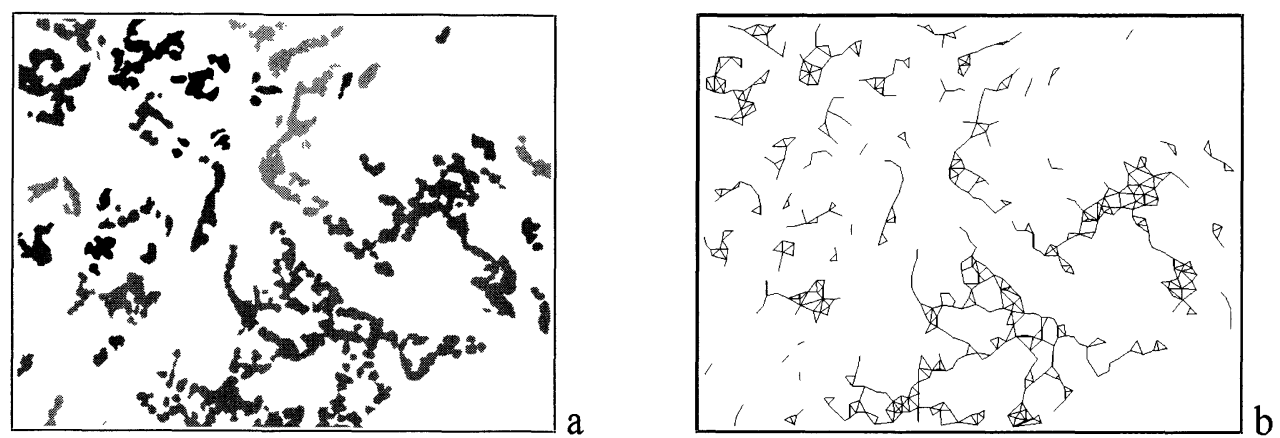

Fig. 7. - Graph segmentation for cluster localization.

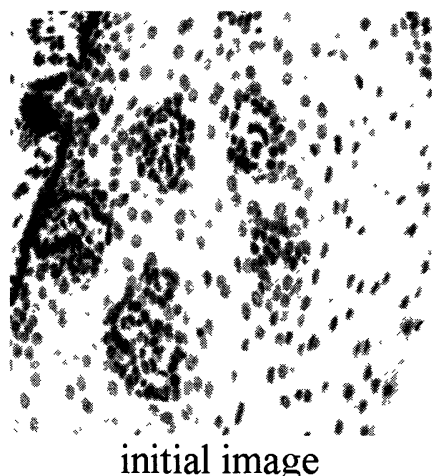

initial image

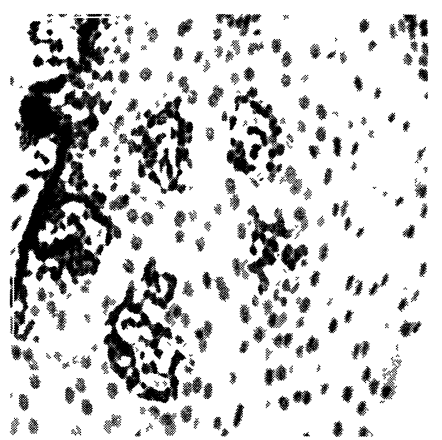

papilla outline

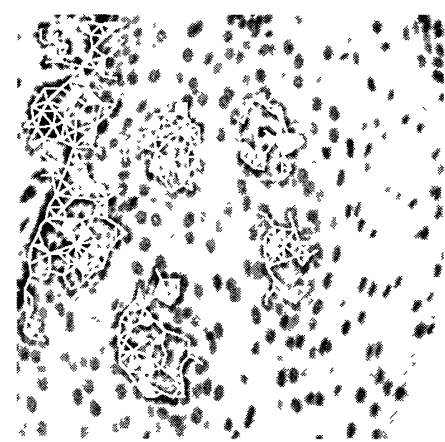

internal structures

Fig. 8. - Example of clusters localization (papilla) in a histological section of oesophageal epithelium.

owing to the fact that graph processing can be used in spaces of any dimension, a 3D library of graph operators (Pandore3D) is being developed, in order to analyse confocal microscopy images.

\section{Acknowledgements}

Our research on graphs is carried out within the frame of the "Pôle Traitement et Analyse d'Images, TAI, de Basse-Normandie". This work is partially supported by a grant from the Ministry of University Education and Research. Special thanks to C. Porquet for her assistance during the translation of the paper.

\section{References}

[1] Chang Y.-L. and Li X., Fast image region growing, Image Vis. C 13 (9/1995) 559-571.

[2] Angot F., Clouard R., Elmoataz A. and Revenu M., Neighbourhood graphs and image processing, "Vision Systems: New Image Processing Techniques", EUROPTO, SPIE 2785 (5/1996) 12-23.

[3] Mazille J.-E., Analyse de structures complexes par la théorie des graphes, La Revue de Métallurgie CIT / Science et Génie des Matériaux 2 (1994) 223-232. 
[4] Marcelpoil R., Usson Y. and Chassery J.-M., Segmentation morphologique incluant des paramètres d'ordre et de désordre : quantification par diagramme de Voronoï et application à la sociologie cellulaire, 8ème Congrès AFCET RFIA, 2 (1992) 967-972.

[5] Raymond E., Raphael M., Grimaud M., Vincent L., Binet J.-L. and Meyer F., Germinal Center Analysis with the Tools of the Mathematical Morphology on Graphs, Cytometry 14 (1993) 848-861.

[6] Voss K., Discrete Images, Objects and Functions in $Z^{\mathrm{n}}$, Algorithms and Combinations 11 (SpringerVerlag, 1993).

[7] Clouard R., Elmoataz A. and Angot F., Pandore, une bibliothèque et un environnement de programmation d'opérateurs de traitement d'images, rapport interne du GREYC, ISMRA (1996).

[8] Vincent L., Graphes et morphologie mathématique, Thèse de Doctorat de l'École des Mines de Paris (1990) chap. 5. 